\title{
An X-ray Free Electron Laser (XFEL) Science Education Model Designed for Large Centers and Its Application To Individual Laboratories
}

\author{
W BAUER ${ }^{1}$ \\ ${ }^{1} \mathrm{HWI}$, Williamsville, NY \\ wbauer@hwi.buffalo.edu
}

The emergence of new technology and techniques involving the use of X-ray Free Electron Lasers (XFEL) has had a profound impact on the advancement of scientific understanding in dynamic systems. These achievements have led to new developments in an array of fields from materials design and watching catalytic reactions occurring in real time to single particle imaging of biomolecules. However, the highly disciplinary field of XFEL science also presents unique challenges in educating the next generation of scientists. Here we present a progressive education program developed by the NSF Science and Technology Center, BioXFEL, and provide specific examples of our initiatives and activities, outcomes and metrics for success. We hope to provide a roadmap and best practices for large, center-scale education programs and individual laboratories that are interested in designing broader impacts for NSF proposals. The Center has created a comprehensive set of customized initiatives that is designed for undergraduate and graduate students, postdoctoral associates and young scientists. Additionally, we routinely lead outreach activities for primary school and high school students. The main educational goal of the Center, at all levels, is to ensure that our scholars are academically and professionally prepared for their current responsibilities and future career aspirations. However, we also recognize that empowering a diverse cohort of future XFEL scientific leaders benefits our scholars as well as the general scientific community. By addressing the specific challenges related to designing and implementing our educational programming model, we hope to provide guidance on creating customized training and professional development opportunities. BioXFEL Science and Technology Center, NSF Award 1231306. Created with input from the BioXFEL Education and Diversity Committee.

Acta Cryst. (2020). A76, a12 